Int. J. Dev. Biol. 51: 379-387 (2007)

doi: $10.1387 / \mathrm{ijdb} .062250 \mathrm{sm}$

Original Article

\title{
Analysis of the role of the Rac/Cdc42 GTPases during planar cell polarity generation in Drosophila
}

\author{
SILVIA MUÑOZ-DESCALZO' ${ }^{1}$, AZUCENA GÓMEZ-CABRERO ${ }^{1}$, MAREK MLODZIK² and NURIA PARICIO*,1 \\ ${ }^{1}$ Department of Genetics, Faculty of Biological Sciences, Valencia University, Spain and ${ }^{2}$ Brookdale Department of Molecular, \\ Cell and Developmental Biology, Mount Sinai School of Medicine, New York, USA.
}

\begin{abstract}
Initial genetic studies in Drosophila suggested that several members of the Rho subfamily (RhoA, Rac1 and Cdc42) are involved in planar cell polarity (PCP) establishment. However, analyses of Rac1, Rac2 and Mt/loss-of-function (LOF) mutants have argued against their role in this process. Here, we investigate in detail the role of the Rho GTPases Mtl, Cdc42, Rac1 and Rac2 in PCP generation. These functional analyses were performed by overexpressing Mtl in eyes and wings, by performing genetic interaction assays and by using a combination of triple and quadruple mutant LOF clones. We found that Mtl overexpression caused PCP phenotypes and that it interacted genetically with other Rho GTPases, such as Rac1 and Cdc42 as well as with several PCP genes, such as stbm, pk and aos. However, Mtl was not found to interact with Rac2, RhoA and other members of the Fz/PCP pathway. Triple mutant clones of Rac1, Rac2 and Mt/ were found to exhibit mild PCP defects which were enhanced by reduction of $C$ dc42function with a hypomorphic Cdc42 allele. Taken together, these and previous results suggest that Rho GTPases may have partially overlapping functions during PCP generation. Alternatively, it is also possible that the mild PCP phenotypes observed could indicate that they are required at low levels in that process. However, since not all of them function upstream of a JNK cassette, we propose that they may act in at least two parallel pathways.
\end{abstract}

KEY WORDS: Mtl, Rac1, Rac2, Cdc42, planar cell polarity, redundancy, Drosophila

\section{Introduction}

Small GTPases act as signal transducers by switching between inactive GDP-bound and active GTP-bound forms and have been implicated in multiple processes during the development of multicellular organisms (Van Aelst and D'SchouzaSchorey, 1997). In Drosophila several members of the Rho subfamily of small GTPases have been identified: Rac1, Rac2, Cdc42, RhoA, RhoL and Mtl (Luo et al., 1994; Harden et al., 1995; Hariharan et al., 1995; Murphy and Montell, 1996; Sasamura et al., 1997; Strutt et al., 1997, Newsome et al., 2000; HakedaSuzuki et al., 2002). Expression of constitutively activated and dominant-negative isoforms of these proteins and analysis of loss-of-function mutants have shed light on their physiological roles. They have been implicated in actin cytoskeleton reorganization (Genova et al., 2000), myogenesis, axonal outgrowth and guidance (Luo etal., 1994; Kaufmann etal., 1998; Hakeda-Suzuki et al., 2002; Ng et al., 2002; Fan et al., 2003), gastrulation (Barrett et al., 1997), oogenesis (Murphy and Montell, 1996), embryonic segmentation (Magie et al., 1999) and cell migration (reviewed by
Montell, 1999; Paladi and Tepass, 2004). They also participate in embryonic dorsal closure (Hakeda-Suzuki et al., 2002; Woolner et al., 2005) and epithelial planar cell polarity establishment (Eaton etal., 1995; 1996; Strutt etal., 1997; Fanto etal., 2000; this paper).

In many organs, epithelial cells are polarized not only along the apical-basolateral axis but also within the plane of the epithelium. The acquisition of this planar cell polarity (PCP) is essential for specialized cellular functions (Klein and Mlodzik, 2005). PCP establishment has emerged as a good model to study the role of Rho family small GTPases. In Drosophila, two members of this family, RhoA and Rac1, have been implicated in this process (Eaton et al., 1995; Strutt et al., 1997; Fanto et al., 2000). PCP phenotypes are characterized by the misorientation of cells within the epithelial plane and have been most extensively studied in the context of Drosophilaeye and wing development. In the eye, PCP is reflected in the mirror-symmetric arrangement of the ommatidia

Abbreviations used in this paper: JNK, Jun N-terminal kinase; LOF, loss-offunction; PCP, planar cell polarity.

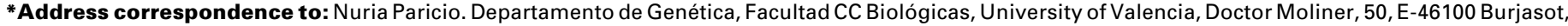
Spain Fax: +34-96-354-3029. e-mail: nuria.paricio@uv.es
} 

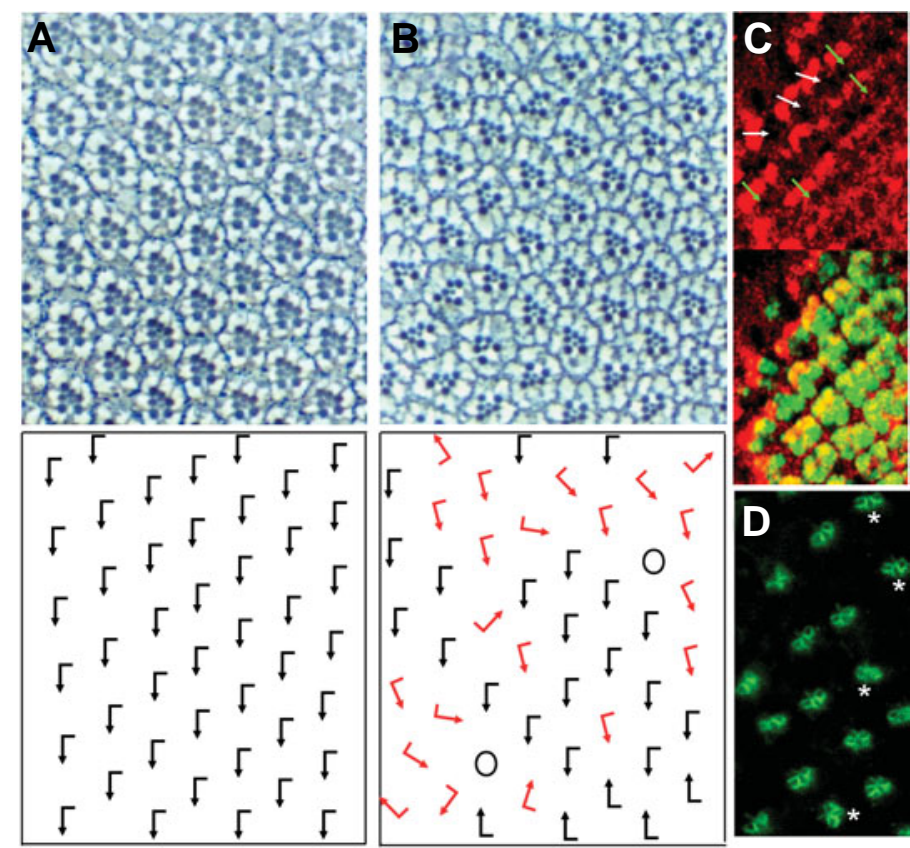

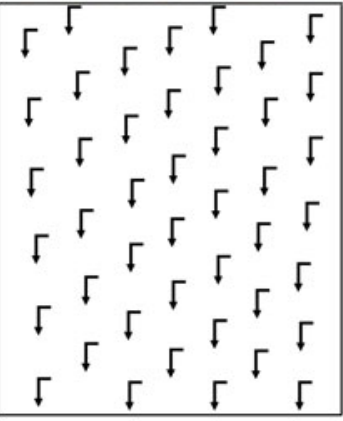

wild type sev $>M t / /+$
Fig. 1. Overexpression of wild-type Mtl causes planar cell polarity (PCP) defects in the eye. (A,B) Tangential sections (upper panels) of adult eyes and the corresponding schematic representation llower panels) with arrows reflecting ommatidial polarity. Anterior is to the left, dorsal is up. Black arrows represent correct ommatidial orientation; red arrows, misrotated ommatidia; circles mark unscorable ommatidia due to missing or malformed photoreceptors. (A) Wild-type eye (dorsal area). (B) sev-GAL4/UAS-Mtl eye. (C) Anti-Spalt staining in third instar larval eye imaginal discS of sev-GAL4/UAS-Mtl flies (upper panel). The lower panel shows overlay of Spalt (red) and anti-Elav (green). White arrows in the red channel mark misoriented ommatidial preclusters and green arrows correctly oriented clusters. (D) Anti-Arm staining in third instar larval eye imaginal discs of sev-GAL4/UAS-Mtl flies. The asterisks show some misoriented ommatidial preclusters.

relative to the dorso-ventral midline, the equator. This pattern is established early in development, when ommatidial preclusters in the dorsal and ventral halves of the disc rotate $90^{\circ}$ in opposite directions. At the same time, they lose their symmetry and opposite chiral forms are established in each half of the eye disc (Mlodzik, 1999, 2002). In the wing, PCP is evident in the uniform pattern formed by distally oriented hairs that cover the dorsal and ventral surfaces. Each wing cell orients itself along the proximal to distal axis and generates a single actin hair pointing distally (Mlodzik, 2002; Eaton, 2003). Mutations in genes that regulate $\mathrm{PCP}$ result in the loss of mirror-image symmetry in the eye, due to failure of ommatidia to acquire the correct chirality and/or to rotate properly. In the wing, PCP defects are manifest in abnormal hair orientation and number of wing hairs per cell.

Genetic and molecular studies have demonstrated that PCP establishment depends on the activity of the Fz/PCP signaling pathway that regulates changes in both cytoskeletal organization and transcription through the JNK pathway (reviewed in Mlodzik, $1999 ; 2002)$. In addition, a conserved group of genes is involved in PCP generation by the formation of multiprotein complexes that are asymmetrically distributed between R3 and R4 (Jenny et al., 2003; Das et al., 2004) and between the proximal and distal wing cell membranes (reviewed in Klein and Mlodzik, 2005). In the eye, Fz/PCP signaling, together with the Notch pathway, is responsible for R3/R4 fate induction and thus for the establishment of ommatidial chirality (Cooper and Bray, 1999; Fanto and Mlodzik, 1999; Tomlinson and Struhl, 1999). Besides, it has been shown that the ommatidial rotation, depends on the Egfr pathway (Brown and Freeman, 2003; Gaengel and Mlodzik, 2003; Strutt and Strutt, 2003). Moreover, downstream of Egfr there is a requirement for the Ras/MAPK cascade and Canoe, an adherensjunction-associated protein (Young et al., 1993), that provides a link from Egfr to cytoskeletal elements (Gaengel and Mlodzik, 2003). Recently, it has been also shown that Egfr signaling regulates Cadherin activity in this context (Mirkovik and Mlodzik, 2006).

Several studies have suggested that the small Rho family GTPases are involved in PCP establishment. RhoA loss-offunction mutants display PCP phenotypes in eyes and wings and they dominantly suppress the gain-of-function phenotypes of $f z$ and $d s h$ (Strutt etal., 1997). Conversely, Cdc42mutants were not found to interact with sev-Fz or sev-Dsh (Boutros et al., 1998), although overexpression of dominant-negative forms of the GTPase in the wing affects actin polymerization during wing hair formation resulting in loss or stunting of hairs (Eaton et al., 1995; 1996), but also producing occasional multiple wing hairs (Baron et al., 2000). The role of Rac in PCP establishment was also addressed using dominant-negative and activated isoforms of Rac1, which produce PCP phenotypes in the eye (Fanto et al., 2000). In addition, deficiencies uncovering either Rac1 or Rac2 dominantly suppress sevE-Dsh (Boutros et al., 1998). However, analyse of loss-of-function mutants in Rac1, Rac2and Mt/did not reveal clear PCP defects in eyes or wings (Hakeda-Suzuki et al., 2002). Mtl is the Drosophila homolog of the C. elegans MIG-2 GTPase (Zipkin et al., 1997; Newsome et al., 2000). Genetic studies of null and gain-of-function mutations of $\mathrm{mig}-2$ in $C$. elegans have shown that this GTPase is required for cell migration and axon guidance and that it functions redundantly with other Rho family GTPases in many cells (Zipkin et al., 1997). Similarly, Mtl is functionally related to Rac1 and Rac2 in Drosophila, because these GTPases act redundantly in regulating

TABLE 1

\section{QUANTIFICATION OF GENETIC INTERACTIONS WITH THE SEV $>M T L$ PHENOTYPE}

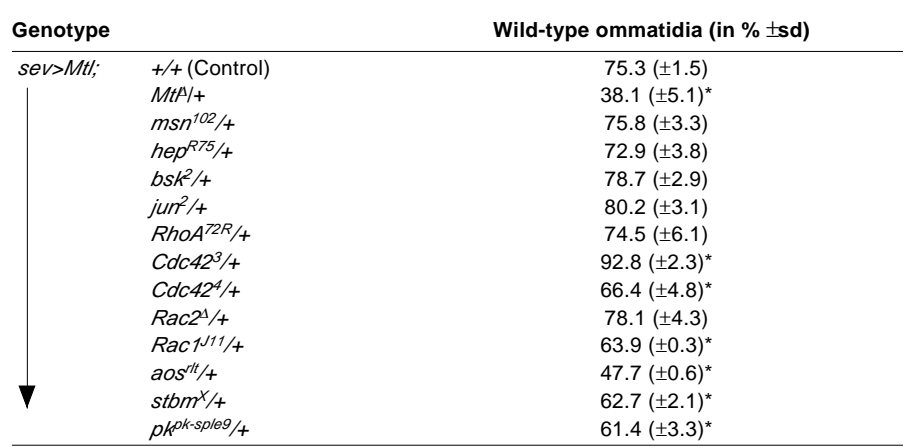

Percentage of wild-type ommatidia ( \pm standard deviation) of the analyzed eyes of flies heterozygous for the indicated alleles and containing one copy of sev>Mt. The quantifications of allelic combinations are based on scoring of 3 to 7 independent eyes per genotype. The asterisks indicate significant interactions $(p \leq 0.05, t$-test). 
dorsal closure and axon growth and guidance (Hakeda-Suzuki et al., 2002, $\mathrm{Ng}$ et al., 2002).

In order to analyze more precisely the requirement of the Rho GTPases Mtl, Rac1, Rac2 and Cdc42 in PCP establishment, we used several strategies. We show that overexpression of wildtype $\mathrm{Mtl}$ in eyes and wings gives rise to PCP defects. Moreover, flies hemizygous for a weak hypomorphic Cdc42 mutant allele also show mild PCP defects in both tissues. In contrast to previous reports (Hakeda-Suzuki et al., 2002), we find that eye clones triple mutant for Rac1, Rac2 and Mt/display mild PCP defects, which are further enhanced when reducing the function of $C d c 42$ with a hypomorphic mutant allele. Taken together, our data suggest that all four Rho GTPases may have a redundant role during PCP generation. Alternatively, it is also possible that the mild PCP phenotypes observed could indicate that they are required at low levels in that process. However, since we find that Mtl and Cdc42 do not act in the canonical Fz pathway, conversely to Rac1, Rac2 and RhoA, we propose that not all the Rho GTPases act upstream of the JNK module and that there are at least two parallel Rho GTPase family functions.

\section{Results}

\section{Overexpression of wild-type Mtl causes PCP phenotypes in eyes and wings}

Functional studies of Rac1, Rac2 and Mtl in Drosophila have shown that they have overlapping functions in the control of epithelial morphogenesis, myoblast fusion, and axon growth and guidance (Hakeda-Suzuki et al., 2002; $\mathrm{Ng}$ et al., 2002). The same study indicates that animals homozygous for a deletion removing the entire $M t /$ open reading frame are fully viable, and suggests that these three GTPases are not required for PCP establishment (Hakeda-Suzuki et al., 2002). However, we have established transgenic flies carrying wild-type $M t /$ under the control of the UAS element (UAS-MtNTlines), and found that overexpression of wildtype $\mathrm{Mtl}$ in eyes and wings using several drivers produce PCP defects. sev-GAL4 drives the expression of $\mathrm{Mtl}$ in the developing eye disc, in the R3/R4 pair, which is critical for establishment of correct polarity. The eyes of the resulting flies ( $\operatorname{sev}>M t N T$, hereafter referred as $\operatorname{sev}>M t$ th are externally rough and reveal typical PCP defects in tangential sections (Fig. 1B). Mtl overexpression resulted in the misorientation of several ommatidia and, at lower frequency, ommatidia with an abnormal complement of photoreceptors. Strikingly, the chirality of the ommatidia was rarely affected and the PCP defects were largely reflected by misrotation (Fig. 1B). As can be seen in Table 1, in sev $>M$ t/eyes $75.3 \%$ of the ommatidia display wild type orientation and the remaining ommatidia show polarity defects $(21 \%)$, mainly misrotation, as well as defects in photoreceptor differentiation (3.7\%). To establish whether the polarity/rotation defects observed with sev $>M t /$ arise early in development and are thus primary defects, we analyzed polarity generation in sev $>M t /$ third instar larval eye imaginal discs (when tissue polarity is first apparent and PCP genes are required). sev $>M t /$ discs were stained with anti-Spalt (as marker for R3/R4 precursors) and anti-Elav (expressed in all photoreceptors). Our results indicate that ommatidial polarity/rotation is affected in sev $>M t /$ eye discs, since the R3/R4 pairs are often incorrectly oriented with respect to their neighbors (Fig. 1C). The misorientation of the photoreceptor clusters is also evident in an anti-Arm staining of sev>Mt/eye discs (Fig. 1D). We also examined the effect of Mtl overexpression in the wing, using the GAL4 lines C765 and en-GAL4. In wild-type wings, each cell produces a single, distally oriented hair (Fig. 2A). Overexpression of Mtl in the whole wing driven by $\mathrm{C} 765$ gave rise exclusively to typical PCP defects and many cells exhibited a multiple wing hair phenotype, producing double or even triple hairs. Moreover, in several areas the wing hairs were not pointing distally, but were misoriented forming waves and whorls (Fig. 2B and data not shown). The same phenotypes were found in the posterior part of adult wings from en-GAL4, UAS-MtNTflies (Fig. 2C). Strikingly, these defects are reminiscent of those of the core PCP genes like $f z$, stbm (also known as vang) or pk (Vinson et al., 1989; Taylor et al., 1998; Gubb et al., 1999).

To test whether the PCP eye phenotype observed in sev $>M t^{\mu T}$ flies is due to excessive Mtl signaling, we tested the effect of reducing the dosage of $M t /$ on such phenotype, using the $M t \AA$ null allele (Hakeda-Suzuki et al., 2002). Surprisingly, we found that the sev $>M t^{N T}$ phenotype is enhanced in an $M t /$ mutant background (Table 1), thus suggesting that this phenotype is not caused by an excessive Mtl signaling but may actually be due to dominantnegative effects. It is possible that the overexpressed Mtl protein may accumulate in an inactive form that could interfere with endogenous Mtl function by directly sequestering it. Alternatively the overexpressed protein could be sequestering or inactivating limiting components or effectors of that GTPase, thus reducing the ability of the cells to signal productively. A similar situation was found when overexpressing the wild type form of Presenilin at very high levels (Ye and Fortini, 1999).

Taken together, our results suggest that $M t /$ has a role in PCP generation in eyes and wings, but it probably functions redundantly
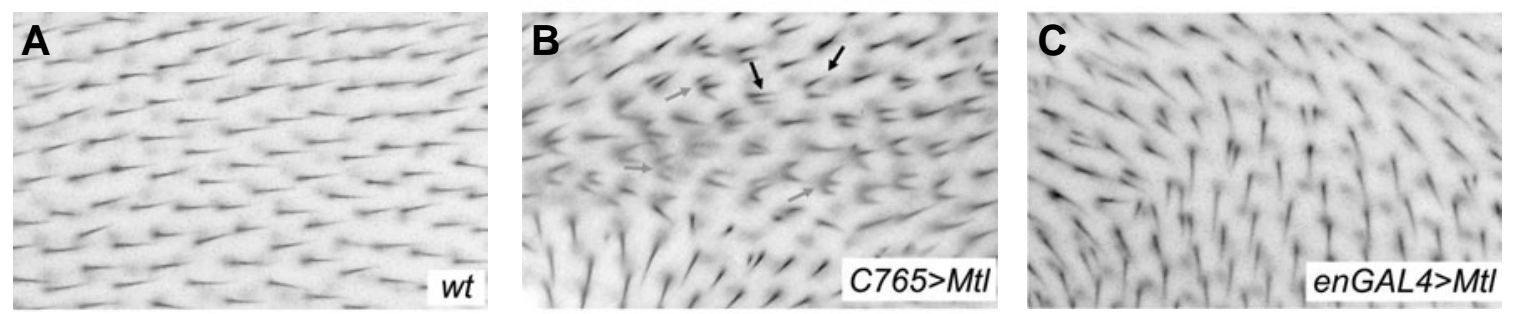

Fig. 2. Overexpression of wild-type $\mathbf{M t}$ c causes $\mathbf{P C P}$ defects in the wing. All panels show high magnification areas of wings, distal is to the right. (A) Wild-type wing. Note the regular arrangement of hairs, all pointing distally. (B) C765/+; UAS-Mtl/+ wing at $25^{\circ} \mathrm{C}$. (C) en-GAL4/+; UAS-Mtl/+ wing at $18^{\circ} \mathrm{C}$ (this cross is lethal when incubated at $25^{\circ} \mathrm{C}$ ). Overexpression of the wild type form of Mtl in the wing causes typical PCP defects, e.g. duplications and triplications of hairs (black and gray arrows, respectively) and misorientation of hairs. Note many hairs pointing perpendicular to the proximal-distal axis in $(B, C)$. 

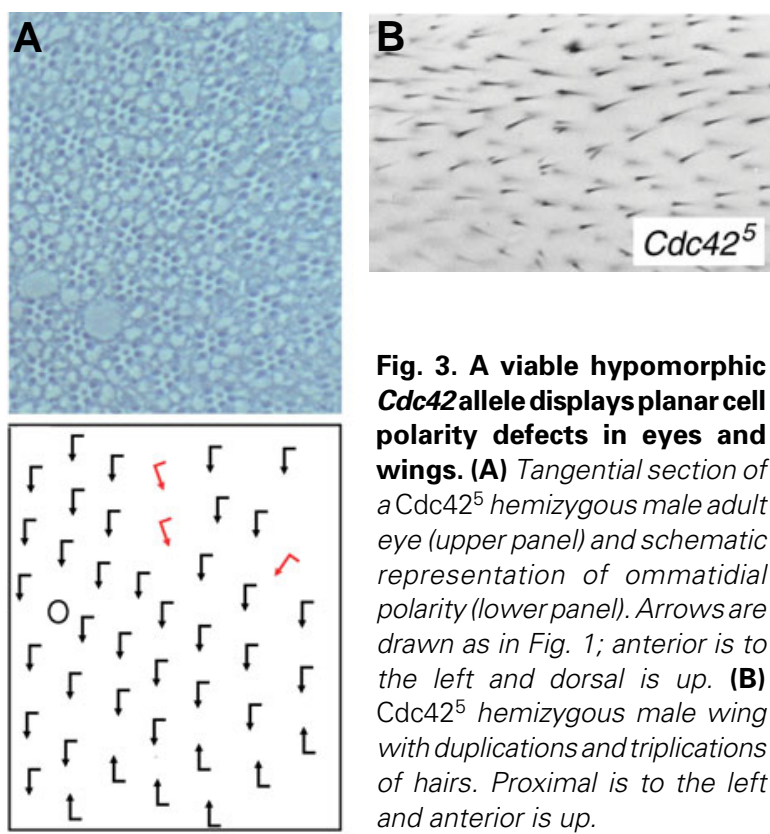

Fig. 3. A viable hypomorphic Cdc42allele displays planar cell polarity defects in eyes and wings. (A) Tangential section of a $\mathrm{Cdc} 42^{5}$ hemizygous male adult eye (upper panel) and schematic representation of ommatidial polarity (lower panel). Arrows are drawn as in Fig. 1; anterior is to the left and dorsal is up. (B) $\mathrm{Cdc} 42^{5}$ hemizygous male wing with duplications and triplications of hairs. Proximal is to the left and anterior is up.

in this process. Since overexpression of wild-type, activated or dominant-negative isoforms of other Rho GTPases like RhoA and Rac1 (Fanto et al., 2000) also results in PCP defects, they could account for the proposed redundancy of function of Mtl during PCP generation.

\section{Mtl does not act in the PCP canonical pathway but interacts genetically with Rac1 and Cdc42}

Since our results suggested that $M t /$ is involved in polarity generation in eyes and wings, we wanted to place it more specifically in the PCP context. To do this we used the sev>Mt/ phenotype to test for genetic interactions with mutations in PCP components and the other GTPases. The results obtained in these experiments are shown in Table 1. Our results indicate that there is no significant genetic interaction between sev>Mt/and the components of the JNK cascade like msn, hep, bsk or jun, suggesting that Mtl does not function upstream of the JNK module. The same result was obtained when reducing the gene dosage of the small GTPase $R$ hoA. Since Mtl is closely related to Rac1 and Rac2, and functionally behaves like both GTPases (Hakeda-Suzuki et al., 2002; $\mathrm{Ng}$ et al., 2002), we also tested for genetic interactions with loss-of-function mutations in both genes ( $R a C 1^{\prime 11}$ and Rac2 $^{21}$, respectively). We found that Rac1, but not Rac2, interacts genetically with the sev>Mt/phenotype. These

\section{TABLE 2}

\section{A NULL MTL MUTANT ALLELE SUPPRESSES THE SEV-RAC1 ${ }^{\text {V12 }}$ PHENOTYPE}

\begin{tabular}{|c|c|c|c|}
\hline Genotype & & $\begin{array}{l}\text { Wild-type ommatidia } \\
\text { (in } \% \pm \mathbf{s d} \text { ) }\end{array}$ & $\begin{array}{c}\text { Number of ommatidia } \\
\text { scored }\end{array}$ \\
\hline sev-Rac1V12; & $\begin{array}{c}+/+(\text { Control }) \\
M t^{A} /+\end{array}$ & $\begin{array}{l}34.1( \pm 4.5) \\
62.3( \pm 3.3)\end{array}$ & $\begin{array}{l}406 \\
389\end{array}$ \\
\hline
\end{tabular}

The quantifications of allelic combinations are based on scoring of 3 independent eyes per genotype. The percentage shown in this table is the average number of wild-type ommatidia, with the standard deviation calculated across all eyes of a given genotype scored. The suppression is statistically significant ( $p \leq 0.01, t$-test). results indicate that $M t /$ might function with Rac1 in the PCP process. Therefore, we wanted to determine whether Mtl and Rac1 act in a hierarchy in the PCP context testing the opposite

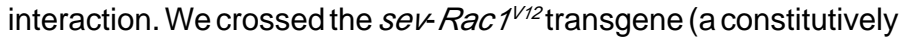
active isoform of Rac1), that produces polarity defects and also interferes with photoreceptor differentiation (Fanto et al., 2000), to the $\mathrm{Mt} /$ /null allele and found that there is a significant suppression of the sev-Rac $1^{1 / 2}$ phenotype (Table 2), reflected in an increase of the number of correctly oriented wild-type ommatidia. Hence, these results support the idea that Mtl functions cooperatively with Rac1, and that they may have a redundant role during PCP establishment.

In addition we have found that $M t /$ genetically interacts with Cdc42. Two lethal alleles were tested for genetic interactions with

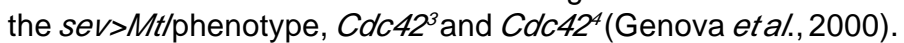
The $C d c 42^{b}$ allele has been used in previous studies which demonstrated that $\mathrm{Cdc} 42$ is not involved in Fz signaling (Boutros et al., 1998), and does not interact with Rac1 (Fanto et al., 2000). We found that both $C d c 42$ mutant alleles dominantly interact with the sev>Mt/phenotype (Table 1), indicating that Cdc42 might be functionally related to $\mathrm{Mtl}$, and suggesting a possible role of Cdc42 in PCP generation (see below). However, while Cdc42 suppressed the sev $>M t /$ phenotype, the $C d c 42^{4}$ allele enhanced it. $C d c 42^{3}$ is a lethal allele in which the conserved Gly residue at position 114 of the protein is replaced by Asp, probably inactivating the Cdc42 protein (Genova et al., 2000). It is interesting to mention that a mutant allele affecting the same region of the $S$. cerevisiae Cdc42 protein was reported to be more than simply a null allele, having a dominant negative effect (Ziman et al., 1991). The $C d c 42^{4}$ mutant contains a nucleotide substitution in an splice acceptor site (Genova et al., 2000), probably producing an incomplete protein and thus reducing Cdc42 function. The results obtained with this allele, which is supposed to be a true loss-offunction allele, suggest that Cdc42 and Mtl may act cooperatively during PCP generation (see below).

\section{Mtl interacts genetically with other genes involved in polarity generation}

We also tested for genetic interactions between $\mathrm{Mt} /$ and other genes involved in PCP establishment like strabismus (stbm, Wolff and Rubin, 1998) and prickle (pk, Gubb et al., 1999). It has been reported that Stbm and Pk are restricted to the R4 precursor cell to properly modulate Fz signaling (Jenny et al., 2003; Rawls and Wolff, 2003). Pk and Stbm interact physically, leading to the assembly of a Stbm/Pk containing signaling complex that is thought to negatively regulate Fz/Dsh activity and membrane localization (Jenny et al., 2003). Both genes dominantly enhance the sev $>M t /$ phenotype (Table 1). One of these genes, stbm, was also found to interact genetically with Rac1 (Fanto et al., 2000). Moreover, we also see a dominant enhancement of this phenotype with the rotation-specific allele of the Egfr-inhibitory ligand argos ( aos $^{\prime / t}$; Gaengel and Mlodzik, 2003). It has been recently reported that Egfr signaling regulates ommatidial rotation through two Raseffector pathways, the Ras/Raf/MAPK cascade and Ras/Cno signaling (Gaengel and Mlodzik, 2003). Moreover, it regulates Cadherin activity in this context (Mirkovik and Mlodzik, 2006). This interaction and the fact that overexpression of Mtl in the eye mainly causes misrotations, suggests an involvement of $\mathrm{Mtl}$ in regulating this aspect of the PCP process. 
TABLE 3

\section{PERCENTAGE OF ABNORMAL OMMATIDIA IN EYE CLONES MUTANT FOR THE INDICATED ALLELIC COMBINATIONS}

\begin{tabular}{|c|c|c|c|}
\hline Genotype & $\begin{array}{l}\text { Abnormal ommatidia } \\
\text { (in } \% \pm \mathbf{s d} \text { ) }\end{array}$ & $\begin{array}{c}\text { Number of clones } \\
\text { analyzed }\end{array}$ & $\begin{array}{c}\text { Number of ommatidia } \\
\text { scored }\end{array}$ \\
\hline $\mathrm{Cdc42^{5 }}$ & $2.9( \pm 3.8)$ & 6 & 422 \\
\hline$R a c \gamma^{111}, \operatorname{Rac}^{2}, M t \wedge \# 1$ & $4.6( \pm 3.1)$ & 7 & 486 \\
\hline $\operatorname{Rac}{ }^{J 11}, \operatorname{Rac}^{2}, M+\AA$ \#2 & $5.5( \pm 2.2)$ & 19 & 1252 \\
\hline 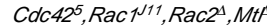 & $14.2( \pm 3.5)$ & 12 & 941 \\
\hline
\end{tabular}

$C d c 42^{5}$ and $R a c 1^{N 11}, R_{a c}{ }^{1}, M t \AA ~ \# 1$ quantifications correspond to control clones generated

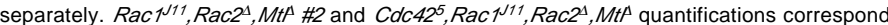
to the results obtained for two populations of different clones obtained from the same cross. The difference in the frequency of abnormal ommatidia between $C d c 42^{5}, \operatorname{Rac}^{N 11}, \operatorname{Rac}^{1}, M+\mathrm{M}^{\mathrm{A}}$ and Rac ${ }^{J 11}, \operatorname{Rac}^{2}, M t \AA \# 1$ or \#2 is statistically significant ( $p \leq 0.001, t$ test). In all cases about $1 \%$ of ommatidia show problems in photoreceptor differentiation.

\section{Cdc42 ${ }^{5}$ mutant flies exhibit mild PCP defects in eyes and wings}

The role of Cdc42 in PCP establishment in the wing has been previously assessed by ectopic expression of different dominant negative isoforms (Eaton et al., 1995; 1996; Baron et al., 2000). These studies suggested that $\mathrm{Cdc} 42$ is necessary for the formation of polarized actin structures, since overexpression of $\mathrm{Cdc} 42^{\mathrm{F} 89}$ or Cdc $42^{\mathrm{L} 89}$ caused abolishment of both actin polymerization and hair outgrowth, resulting in wings with no hair or with stunted hairs (Eaton et al., 1995; 1996). In addition, expression of Cdc42 ${ }^{\mathrm{N} 17}$ produced a multiple wing hair phenotype (Baron et al., 2000). In the eye, it has been demonstrated that flies heterozygous for weak and strong Cdc42 mutant alleles have mild rough eyes, although no defects in ommatidial orientation were reported (Boutros etal., 1998; Genova et al., 2000).

To test more definitively whether Cdc42 plays a role in PCP establishment, we analyzed the effect of reducing Cdc42 function in eyes and wings. As clones of the null allele $C d c 42^{3}$ and $C d c 42^{4}$ do not survive (Genova et al., 2000; data not shown), we analyzed tangential sections of eyes from $\mathrm{Cdc} 2^{5}$ (a hypomorphic allele) hemizygous males. These show PCP defects, although at very low frequency (2.9\%; Table 3$)$, reflected by the presence of misrotated and achiral ommatidia (Fig. $3 A$ and data not shown). Similar results have been found in females in homozygous mitotic eye clones for the $\mathrm{Cdc}_{4} 2^{5}$ allele (Table 3). Moreover, analyses of wings from Cdc $42^{5}$ individuals also revealed mild PCP defects, with cells exhibiting a multiple wing hair phenotype, producing double or triple hairs (Fig. 3B). These data suggest that $C d c 42$ has a role in PCP generation in eyes and wings, and could account for the redundancy of function proposed for the Racs and Mtl in this process. In support of this model, a study has demonstrated that Cdc42 acts redundantly with Rac1 and Rac2 during embryonic blood cell migration (Paladi and Tepass, 2004).

\section{Eye clones mutant for different combinations of Mtl, Rac1, Rac2 and Cdc42 alleles display polarity phenotypes}

To further investigate the role of Mtl, Rac1, Rac2and Cdc42in PCP establishment, we generated eye clones mutant for different allelic combinations of all four genes. We used null alleles for Rac1, Rac2 and Mt/ (Rac1 ${ }^{111}$, Rac2 ${ }^{1}$ and MtA, respectively) and the hypomorphic $C d c 42^{5}$ allele. Phenotypic analyses of eye clones double mutant for either Rac1 and Mt/or Rac1 and Rac2 revealed no PCP defects (data not shown). Besides, clones mutant for either Mt/and Cdc42or Rac1, Rac2and Cdc42showed a low frequency of PCP defects (data not shown), comparable to the results obtained in $C d c 42^{5}$ mutants or mutant clones. Next we generated eye clones triply mutant for Rac1, Rac2 and Mtl. Although previous analyses of such clones suggested that there is no requirement of these GTPases during PCP generation (Hakeda-Suzuki etal., 2002), we detected reproducible ommatidial polarity defects (Fig. 4A). Analyses of such clones revealed that, although many ommatidia display correct polarity, achiral or misrotated triply mutant ommatidia are reproducibly detected
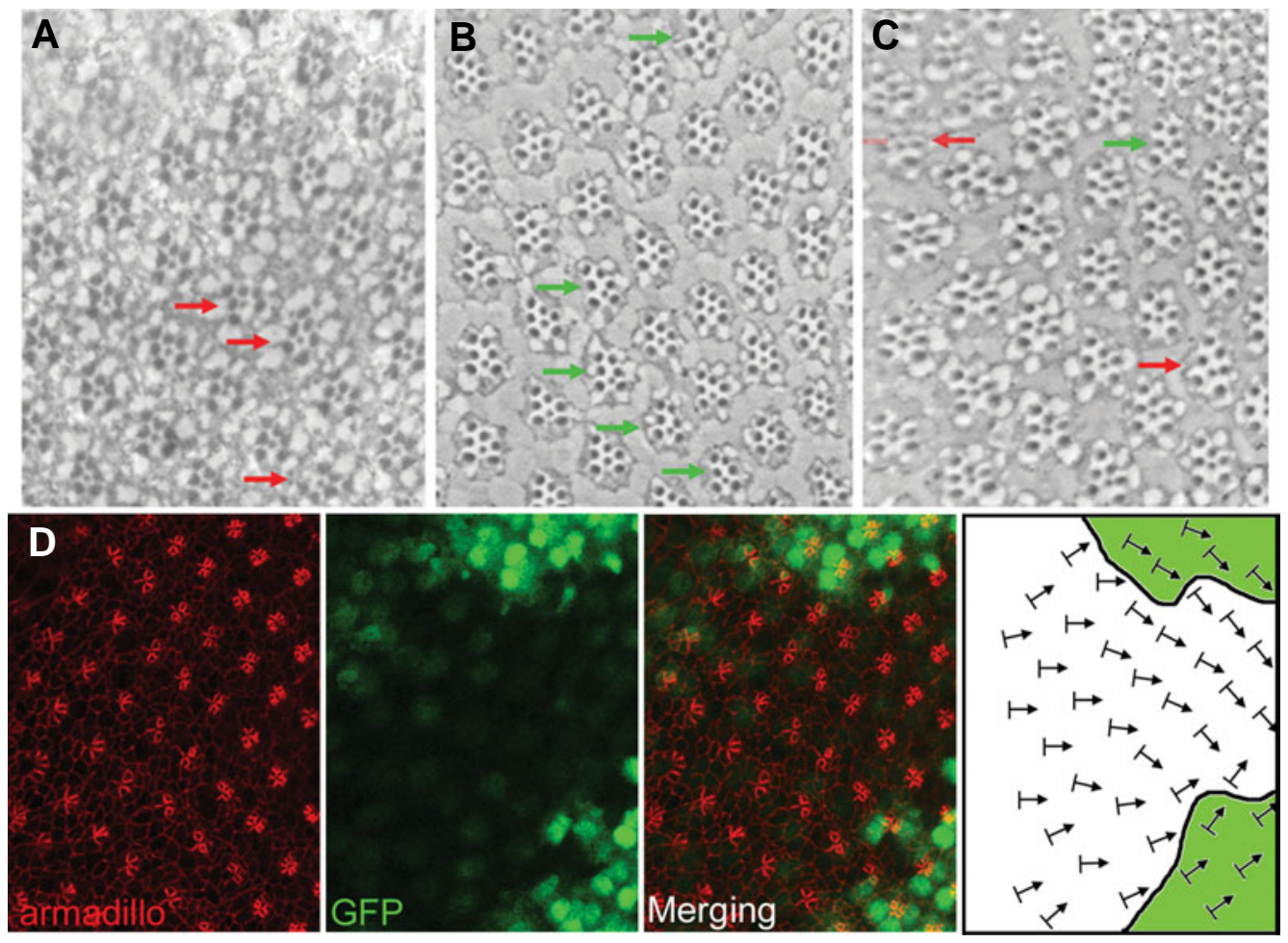

Fig. 4. Eye clones which are quadruple mutant for Rac1, Rac2, MtI and Cdc42 show planar cell polarity defects. (A) Homozygous mutant clone for Rac1 ${ }^{\mathrm{J} 11}$, Rac2 ${ }^{\Delta}$ and $\mathrm{Mt} \mathrm{I}^{\Delta}$. (B,C) Clones homozygous mutant for $\mathrm{Cdc}_{4} 2^{5}, \mathrm{Rac}^{\mathrm{J} 11}, \mathrm{Rac}^{\Delta}{ }^{\Delta}$ and $\mathrm{Mtl}^{\Delta}$. Red arrows point to misrotated ommatidia, green arrows point to achiral ommatidia. (D) Anti-Arm staining (red) in third instar larval eye imaginal disc with a Cdc42 ${ }^{5}, \mathrm{Rac}^{\mathrm{J} 11}, \mathrm{Rac}^{\Delta}$ and $\mathrm{Mtl}^{\Delta}$ homozygous mutant clone (marked by the absence of GFP fluorescence). The last panel is a schematic representation of the ommatidial rotation angles in the clone. 
(4.6\% of abnormal ommatidia, see Rac ${ }^{N 11}$, Rac2 , Mt/ \#1 in Table 3). The low penetrance of this phenotype may indicate that the Rac genes play a minor role during PCP generation. However, it can also suggest that their function is largely redundant in this process. Since we have found that $\mathrm{Cdc} 42$ also has a role during PCP generation, we asked whether the PCP defects found in eye clones triply mutant for the three GTPases Rac1, Rac2 and Mt/ could be modified by reducing the function of $\mathrm{Cdc} 42$. We thus generated clones that were quadruple mutant for all four GTPases (see Material and Methods). Strikingly, these quadruple mutant clones display PCP defects at higher frequency. Analysis of tangential sections of such clones revealed typical PCP defects, like symmetrical and misrotated ommatidia (Fig. 4B,C), with a frequency of close to 15\% (Table 3, see $C d c 42^{5}$, Rac ${ }^{111}$, Rac2 $2^{1}$, MtA ). Control clones triply mutant for the three Rac genes (originating from the same cross) showed defects at $5.5 \%$ frequency, comparable to the $4.6 \%$ obtained in the original triply mutant clones (Table 3, compare Rac ${ }^{111}$, Rac2 ${ }^{1}$, MtA \#2 to $\operatorname{Rac}{ }^{111}, \operatorname{Rac}^{1}$, MtP \#1). This indicates that the reduction of $C d c 42$ function is causing the increased PCP defects and that these are not due to genetic background variation in the clones. Armadillo stainings of quadruple mutant eye disc clones confirmed rotation abnormalities and revealed no loss of accessory ommatidial cells that could lead to problems in local cell stacking (Fig. 4D). To support our results in the eye, we have also generated unmarked quadruple mutant clones in adult wings. In these wings we occasionally observed PCP phenotypes, like duplications of wing hairs and groups of hairs that were not pointing distally (data not shown).

Taken together, these results could indicate that the Rac/ Cdc42 GTPases may have overlapping functions during PCP establishment in the Drosophila eye. Then, the low penetrance of the mutant phenotype in the quadruple mutant clones, which is

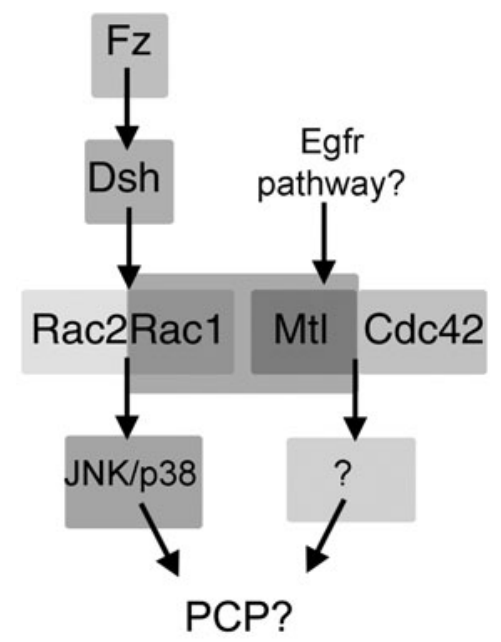

Fig. 5. GTPases of the Rho subfamily function in parallel pathways. A simplified schematic view of the Fz/PCP pathway and the relative position of several members of the Rho subfamily of GTPases are shown. As was previously reported, Rac1 and Rac2 lead to transcriptional activation through the JNK/p38s MAPK cascade. In addition, Mtl might function in the Egfr pathway. However, the specific effectors of $\mathrm{Mtl} / \mathrm{Cdc} 42$ are currently unknown. comparable to hypomorphic alleles of PCP genes, would be consistent with $C d c 42^{5}$ being a hypomorphic allele. However, another explanation for the mild PCP phenotypes observed in the clones could be that these proteins have a minor role during the PCP process. As mentioned above, the closely related RhoA GTPase plays a non-redundant role in PCP establishment (Strutt etal., 1997). One possibility could be that the Rac/Cdc42 GTPases cooperate with RhoA during PCP generation. Then, a reduction of $R$ hoAfunction could also modify the PCP defects obtained by loss of function of the Rac genes. To test this, we generated clones quadruple mutant for the Rac1, Rac2, Mt/and RhoA genes (using the strong hypomorphic allele $R h o A^{A Y}$; Strutt etal., 1997). However, these quadruple mutant clones were not informative as we found mainly photoreceptor loss in such clones, obscuring a potential to score for PCP defects (data not shown). Since the proposed redundancy among the Rho GTPases during PCP generation has not been demonstrated, we can not rule out the possibility that these proteins are required at low levels during this process.

\section{Discussion}

In this report we have analyzed in detail the role of Mtl, Rac1, Rac2 and Cdc42, four GTPases of the Rho subfamily in Drosophila. First, we show that overexpression of different Mtl isoforms in eyes and wings produces classical PCP phenotypes, as previously reported for other members of the family, thus suggesting that Mtl also has a role during PCP generation in these tissues. Moreover, genetic interaction assays indicate that $\mathrm{Mtl}$ is functionally related to Rac1 and Cdc42, but it does not function in the JNK pathway. Since previous results showed that Rac1 interacts genetically with Rac2 and RhoA (Fanto et al., 2000), we conclude that Rac1, Rac2 and RhoA could act redundantly in Fz/PCP signaling, and Mtl could be acting together with $\mathrm{Cdc} 42$, both aspects being connected through Rac1 and Mtl. Regarding this, we also show that flies hemizygous for a hypomorphic Cdc42allele, as well as mitotic eye clones for the same allele, display typical PCP defects, thus suggesting that Cdc42 may also function in PCP generation. In such a scenario, and in contrast to previously published results (Hakeda-Suzuki et al., 2002), we also demonstrate that mitotic eye clones triply mutant for the Rac GTPases (Mtl, Rac1 and Rac2) show polarity defects, albeit at relatively low frequency. Strikingly, the frequency of the defects is increased in the triple null Rac mutant background by reducing $C d c 42$ function with a $C d c 42$ hypomorphic allele. Taken together, all these results suggest that the Rac/Cdc42 GTPases may have a role during PCP generation but probably function redundantly in this process, since only the removal of the four GTPases causes PCP defects. An explanation for the mild PCP phenotypes observed in the quadruple mutant clones, which is comparable to hypomorphic alleles of PCP genes, would be the fact that $C d c 42^{5}$ is a hypomorphic allele. In such a scenario, RhoA, another small GTPase of the Rho subfamily, has a well established and non-redundant role in this process (Strutt et al., 1997), and it interacts genetically with Rac1 (Fanto et al., 2000). Since there is a high degree of homology among all these proteins, one possibility could be that the Rac/Cdc42 GTPases cooperate with RhoA during PCP generation. However, since we could not demonstrate the proposed redundancy of function between all these GTPases, an alternative explanation could be that the Rac/Cdc42 GTPases are required at low levels during PCP establishment. 
Besides this, the results obtained in the genetic interaction assays indicate that not all the GTPases of the Rho subfamily function upstream the JNK module and that they act in parallel pathways. Our results and previous reports suggest that although Rac1 and Rac2 function downstream of Dsh in the Fz/PCP pathway through JNK/p38 kinases (Boutros et al., 1998; Paricio et al., 1999; Weber et al., 2000), Mtl and Cdc42 might receive a different activating input, as they do not show genetic interactions with gain-of-function $F z$ and Dsh phenotypes, and Mtl does not interact with JNK components (Boutros et al., 1998; this paper). It is interesting to mention that the rotation-specific phenotype obtained by $\mathrm{Mtl}$ overexpression, together with the fact that this GTPase genetically interacts with members of the Egfr pathway and cell adhesion components related to it (data not shown), could indicate that Mtl function in the Egfr pathway regulating ommatidial rotation during the final steps of PCP establishment (this paper; F. Durupt, S.M.-D. and N.P., in preparation). Taken together, all these observations suggest that the requirement of the four GTPases might be subdivided into pairs: Mtl could share a function with Cdc42 (this is supported by data from mammalian tissue culture experiments, where Cdc42 and mammalian Mtl appear to have the same function; A. Hall, personal communication) and Rac1 with Rac2, and both pairs would be connected through the shared functional Rac1-Mtl interaction (Fig. 5). However, whether the GTPase pairs (Mtl-Cdc42, Rac1-Rac2, and Rac1-Mtl) act in parallel or in a hierarchy remains unclear. Although we did not include RhoA in our model, this GTPase functions downstream of Dsh in the Fz/PCP pathway (Strutt et al., 1997) and upstream of the JNK cassette, and interacts genetically with Rac1 (Fanto et al., 2000). Thus, it will function together with the Rac1-Rac2 pair.

In summary, our results are similar to the data obtained from the study of vertebrate gastrulation. Habas et al. (2003) have reported that RhoA and Rac have independent parallel roles during the convergent extension process in vertebrate gastrulation downstream of Fz-Dsh signaling, and that only Rac is able to activate JNK. This is consistent with our data (and previous publications on Drosophila PCP generation; Eaton et al., 1996; Fanto et al., 2000). In addition, our data suggest that Cdc42could function redundantly with the Rac genes. Supporting this, Cdc42 has also a reported role in convergent extension/vertebrate gastrulation (Choi and Han, 2002). Whether and how this is linked to Fz-PCP signaling remains unclear. Although our results do not provide clear evidence of the redundant function of the GTPases of the Rho subfamily during PCP generation, we can conclude that the situation in vertebrates and Drosophila is similar: not all the GTPases act upstream of a JNK cassette, and there are probably (at least) two parallel Rho GTPase family functions.

\section{Materials and Methods}

\section{Generation of flies expressing Mtl transgenes}

To generate the UAS-Mt/wild type construct, the complete Mt/cDNA was cloned into the pUAST Drosophilatransformation vector (Brand and Perrimon, 1993). Transgenic flies were generated by standard P-element-mediated transformation (Spradling and Rubin, 1982).

\section{Fly strains and genetic interactions}

Flies were grown on standard media at $25^{\circ} \mathrm{C}$ (unless stated otherwise). GAL4 stocks used were: sev-GAL4 K25 for the third chromosome (gift from Konrad Basler), en-GAL4 and C765-GAL4. Mutant stocks used were: $m s n^{102}$ (Treisman et al., 1997), RhoA ${ }^{72 R}$, RhoA $A^{A Y}$ (Strutt et al., 1997), hep ${ }^{R 75}$ (Glise etal., 1995), bsk (Riesgo-Escovar etal., 1996), jun ${ }^{2}$ (Kockel et al., 1997), stbm ${ }^{X}$ (N. Paricio, unpublished), pkpk-spleg (Gubb et al., 1999), aos ${ }^{\text {rt }}$ (Gaengel and Mlodzik, 2004), Rac111, Rac24, MtA (Hakeda-Suzuki etal., 2002), Cdc42 $2^{3}, C d c 42^{4}$ and $C d c 42^{5}$ (Genova etal., 2000).

We also used the sevenlessenhancer driven construct sev-Rac ${ }^{V 12}$ for interactions with $M t /$ alleles (Fanto et al., 2000). A sev $>M t /$ line was generated by recombination of sev-GAL4 and UAS-Mt/ chromosomes. Genetic interactions with that line were performed at $25^{\circ} \mathrm{C}$. The flies analyzed were heterozygous for sev $>M t /$ and the mutation of interest. $w^{1118}$ was used as a negative control.

\section{Generation of mitotic eye clones}

Eye clones were generated with the FRT/FLP recombination system (Golic and Linquist, 1989) using ey-FLP lines. To generate clones quadruple mutant for Rac1, Rac2, Mt/and Cdc42we set up two independent crosses. First, $C d c 42^{5}$,FRT19A females were crossed to Racyl11 Rac2 ${ }^{4}, M^{4}{ }^{4}, \mathrm{FRT} 2 \mathrm{~A} / \mathrm{TM} 6$ (Hakeda-Suzuki et al., 2002) males. Simultaneously, we crossed FRT19A/FM6;ey-FLP females to ey-FLP/Y;spl CyO;2x UbiGFP,FRT2A/TM6, Ubx males. From both, we selected the non-balanced progeny and crossed non-balanced males from the first cross to non-balanced females from the second cross. The eye clones are marked by the absence of pigment in adults and by the absence of GFP fluorescence in discs. Adult eye clones were analyzed only in the female offspring, in which $50 \%$ will be quadruple mutant for Rac1, Rac2, Mt/and Cdc42 and $50 \%$ will be triply mutant for Rac1, Rac2 and Mtl. Among the clones analyzed, we could distinguish two different populations, based on the frequency of the PCP defects they contained. One population exhibited a similar frequency of defects than the control triple mutant clones generated and corresponds to clones mutant only for the Racgenes. The second population showed PCP defects at higher frequency than the controls and corresponds to the quadruple mutant clones. The differences in frequency of defects between both populations are statistically significant.

\section{Histology and immunohistochemistry}

Sections of adult eyes were performed as previously described (Tomlinson and Ready, 1987). Wings were dissected from adult flies in $\mathrm{SH}$ solution and mounted in Faure medium. Imaginal disc stainings were done in $0.1 \mathrm{M}$ phosphate buffer, $0.2 \%$ Triton $\mathrm{X}-100$ and $10 \%$ normal goat serum. Primary antibodies used were mouse anti-Elav and mouse antiArmadillo (both from the Developmental Studies Hybridoma Bank), rabbit anti-Spalt (gift from Rosa Barrio) and rabbit anti- $\beta$-gal (polyclonal from Cappel). Secondary antibodies coupled to fluorochromes were purchased from Calbiochem. Pictures were taken using a Leica TCS-NT confocal laser-scanning microscope.

\section{Acknowledgements}

We are grateful to $R$. Barrio for the anti-Spalt antibody. We thank $L$. Luo, B. Dickson and the Szeged and Bloomington Stock Centers for fly strains and $A$. Hall for sharing unpublished observations. Confocal microscopy was performed at the SCSIE (Universitat de València). S. M.D. was supported by a fellowship from Consellería de Cultura, Educació i Ciència. This work has been supported by Consellería de Cultura, Educació i Ciència, Ministerio de Educación y Ciencia and NIH/NEl.

\section{References}

BARON, M., O'LEARY, V., EVANS, D.A., HICKS, M. and HUDSON, K. (2000). Multiple roles of the Dcdc42 GTPase during wing development in Drosophila melanogaster. Mol. Gen. Genet. 264: 98-104.

BARRETT, K., LEPTIN, M. and SETTLEMAN, J. (1997). The Rho GTPase and a putative RhoGEF mediate a signaling pathway for the cell shape changes in Drosophila gastrulation. Cel/91: 905-915. 
BOUTROS, M., PARICIO, N., STRUTT, D.I. and MLODZIK, M. (1998). Dishevelled activates JNK and discriminates between JNK pathways in planar polarity and wingless signaling. Ce//94: 109-118.

BRAND, A.H. and PERRIMON, N. (1993). Targeted gene expression as a means of altering cell fates and generating dominant phenotypes. Dev. Biol. 42: 211221.

BROWN, K.E. and FREEMAN, M. (2003). Egfr signalling defines a protective function for ommatidial orientation in the Drosophila eye. Development 130: 5401-5412.

CHOI, S.C. and HAN, J.K. (2002). Xenopus Cdc42 regulates convergent extension movements during gastrulation through $\mathrm{Wnt} / \mathrm{Ca} 2+$ signaling pathway. Dev. Biol. 244: 342-357.

COOPER, M.T. and BRAY, S.J. (1999). Frizzled regulation of Notch signalling polarizes cell fate in the Drosophila eye. Nature 397: 526-530.

DAS, G., JENNY, A., KLEIN, T.J., EATON, S. and MLODZIK, M. (2004). Diego interacts with Prickle and Strabismus/Van Gogh to localize planar cell polarity complexes. Development 131: 4467-4476.

EATON, S. (2003). Cell biology of planar polarity transmission in the Drosophila wing. Mech. Dev. 120: 1257-1264.

EATON, S., AUVINEN, P., LUO, L., JAN, Y.N. and SIMONS, K. (1995). CDC42 and Rac1 control different actin-dependent processes in the Drosophila wing disc epithelium. J. Cell Biol. 131: 151-164.

EATON, S., WEPF, R. and SIMONS, K. (1996). Roles for Rac1 and Cdc42 in planar polarization and hair outgrowth in the wing of Drosophila. J. Cell Biol. 135: 12771289.

FAN, X., LABRADOR, J.P., HING, H. and BASHAW, G.J. (2003). Slit stimulation recruits Dock and Pak to the roundabout receptor and increases Rac activity to regulate axon repulsion at the CNS midline. Neuron 40: 113-127.

FANTO, M. and MLODZIK, M. (1999). Asymmetric Notch activation specifies photoreceptors R3 and R4 and planar polarity in the Drosophila eye. Nature 397: 523-526.

FANTO, M., WEBER, U., STRUTT, D.I. and MLODZIK, M. (2000). Nuclear signaling by Rac and Rho GTPases is required in the establishment of epithelial planar polarity in the Drosophila eye. Curr. Biol. 10: 979-988.

GAENGEL, K. and MLODZIK, M. (2003). Egfr signaling regulates ommatidial rotation and cell motility in the Drosophila eye via MAPK/Pnt signaling and the Ras effector Canoe/AF6. Development 130: 5413-5423.

GENOVA, J.L., JONG, S., CAMP, J.T. and FEHON, R.G. (2000). Functional analysis of $C d c 42$ in actin filament assembly, epithelial morphogenesis and cell signaling during Drosophila development. Dev. Biol. 221: 181-194.

GLISE, B., BOURBON, H. and NOSELLI, S. (1995). hemipterous encodes a novel Drosophila MAP kinase kinase, required for epithelial cell sheet movement. Cell 83: 451-461.

GOLIC, K.G. and LINQUIST, S. (1989). The FLP recombinase of yeast catalizes site-specific recombination in the Drosophila genome. Ce//59: 499-509.

GUBB, D., GREEN, C., HUEN, D., COULSON, D., JOHNSON, G., TREE, D., COLLIER, S. and ROOTE, J. (1999). The balance between isoforms of the prickle LIM domain protein is critical for planar polarity in Drosophila imaginal discs. Genes Dev. 13: 2315-2327.

HABAS, R., DAWID, I.B. and HE, X. (2003). Coactivation of Rac and Rho by Wnt/ Frizzled signaling is required for vertebrate gastrulation. Genes Dev. 17: 295309.

HAKEDA-SUZUKI, S., NG, J., TZU, J., DIETZL, G., SUN, Y., HARMS, M., NARDINE, T., LUO, L. and DICKSON, B.J. (2002). Rac function and regulation during Drosophila development. Nature 416: 438-442.

HARDEN, N., LOH, H.Y., CHIA, W. and LIM, L. (1995). A dominant version of the small GTP-binding protein Rac disrupts cytoskeletal structures and inhibits developmental cell shape changes in Drosophila. Development 121: 903-914.

HARIHARAN, I.K., HU, K.Q., ASHA, H., QUINTANILLA, A., EZZELL, R.M. and SETTLEMAN, J. (1995). Characterization of rho GTPase family homologues in $D$. melanogaster. overexpressing Rho1 in retinal cell causes a late developmental defect. EMBO J. 14: 292-302.

JENNY, A., DARKEN, R.S., WILSON, P.A. and MLODZIK, M. (2003). Prickle and Strabismus form a functional complex to generate a correct axis during planar cell polarity signaling. EMBO J. 22: 4409-4420.
KAUFMANN, N., WILLS, Z.P. and VAN VACTOR, D. (1998). Drosophila Rac1 controls motor axon guidance. Development 125: 453-461.

KLEIN, T.J. and MLODZIK, M. (2005). Planar Cell Polarization: An Emerging Model Points in the Right Direction. Annu. Rev. Cell Dev. Biol. 21: 155-176.

KOCKEL, L., ZEITLINGER, J., STASZEWSKI, L.M., MLODZIK, M. and BOHMANN, D. (1997). Jun in Drosophila development: redundant and nonredundant functions and regulation by two MAPK signal transduction pathways. Genes Dev. 11: $1748-1758$

LUO, L., LIAO, Y.J., JAN, L.Y. and JAN, Y.N. (1994). Distinct morphogenetic functions of similar small GTPases: Drosophila Drac1 is involved in axonal outgrowth and myoblast fusion. Genes Dev. 8: 1787-1802.

MAGIE, C.R., MEYER, M.R., GORSUCH, M.S. and PARKHURST, S.M. (1999). Mutations in the Rho1 small GTPase disrupt morphogenesis and segmentation during early Drosophila development. Development 126: 5353-5364.

MIRKOVIK, I. and MLODZIK, M. (2006). Cooperative activities of Drosophila DECadherin and DN-Cadherin regulate the cell motility process of ommatidial rotation. Development 133: 3283-3293.

MLODZIK, M. (1999). Planar polarity in the Drosophila eye: a multifaceted view of signaling specificity and cross-talk. EMBO J. 24: 6873-6879.

MLODZIK, M. (2002). Planar cell polarization: do the same mechanisms regulate Drosophila tissue polarity and vertebrate gastrulation? Trends Genet. 18: 564 571.

MONTELL, D.J. (1999). The genetics of cell migration in Drosophila melanogaster and Caenorhabditis elegans development. Development 126: 3035-3046.

MURPHY, A.M. and MONTELL, D.J. (1996). Cell type-specific roles for Cdc42, Rac and RhoL in Drosophila oogenesis. J. Cell Biol. 133: 617-630.

NEWSOME, T.P., SCHMIDT, S., DIETZL, G., KELEMAN, K., ASLING, B., DEBANT, A. and DICKSON, B.J. (2000). Trio combines with dock to regulate Pak activity during photoreceptor axon pathfinding in Drosophila. Cel/101: 283-294.

NG, J., NARDINE, T., HARMS, M., TZU, J., GOLDSTEIN, A., SUN, Y., DIETZL, G. DICKSON, B.J. and LUO, L. (2002). Rac GTPases control axon growth, guidance and branching. Nature 416: 442-447.

PALADI, M. and TEPASS, U. (2004). Function of Rho GTPases in embryonic blood cell migration in Drosophila. J. Cell Sci. 117: 6313-6326.

PARICIO, N., FEIGUIN, F., BOUTROS, M., EATON, S. and MLODZIK, M. (1999). The Drosophila STE20-like kinase misshapen is required downstream of the Frizzled receptor in planar polarity signalling. EMBO J. 18: 4669-4678

RAWLS, A.S. and WOLFF, T. (2003). Strabismus requires Flamingo and Prickle function to regulate tissue polarity in the Drosophila eye. Development 130: 1877-1887.

RIESGO-ESCOVAR, J.R., JENNI, M., FRITZ, A. and HAFEN, E. (1996). The Drosophila Jun-N-terminal kinase is required for cell morphogenesis but not for DJun-dependent cell fate specification in the eye. Genes Dev. 10: 2759-2768.

SASAMURA, T., KOBAYASHI, T., KOJIMA, S., QADOTA, H., OHYA, Y., MASAI, I. and HOTTA, Y. (1997). Molecular cloning and characterization of Drosophila genes encoding small GTPases of the rab and rho families. Mol. Gen. Genet. 254: 486-494.

SPRADLING, A.C. and RUBIN, G.M. (1982). Transposition of cloned P-elements into Drosophila germ line chromosomes. Science 218: 341-347.

STRUTT, H. and STRUTT, D. (2003). EGF signaling and ommatidial rotation in the Drosophila eye. Curr. Biol. 13: 1451-1457.

STRUTT, D.I., WEBER, U. and MLODZIK, M. (1997). The role of RhoA in tissue polarity and Frizzled signalling. Nature 387: 292-295.

TAYLOR, J., ABRAMOVA, N., CHARLTON, J. and ADLER, P.N. (1998). Van Gogh: a new Drosophila tissue polarity gene. Genetics 150: 199-210.

TOMLINSON, A. and READY, D.F. (1987). Neuronal differentiation in the Droso phila ommatidium. Dev. Biol. 120: 366-376

TOMLINSON, A. and STRUHL, G. (1999). Decoding vectorial information from a gradient: sequential roles of the receptors Frizzled and Notch in establishing planar polarity in the Drosophila eye. Development 126: 5725-5738.

TREISMAN, J.E., ITO, N. and RUBIN, G.M. (1997). misshapen encodes a protein kinase involved in cell shape control in Drosophila. Gene 186: 119-125.

VAN AELST, L. and D'SCHOUZA-SCHOREY, C. (1997). Rho GTPases and signaling networks. Genes Dev. 11: 2295-2322. 
VINSON, C.R., CONOVER, S. and ADLER, P.N. (1989). A Drosophila tissue polarity locus encodes a protein containing seven potential transmembrane domains. Nature 338: 263-264

WEBER, U., PARICIO, N. and MLODZIK, M. (2000). Jun mediates Frizzled-induced R3/R4 cell fate distinction and planar polarity determination in the Drosophila eye. Development 127: 3619-3629.

WOLFF, T. and RUBIN, G.M. (1998). strabismus, a novel gene that regulates tissue polarity and cell fate decisions in Drosophila. Development 125: 1149-1159.

WOOLNER, S., JACINTO, A. and MARTIN, P. (2005). The small GTPase Rac plays multiple roles in epithelial sheet fusion-dynamic studies of Drosophila dorsal closure. Dev. Biol. 282: 163-173.

YE, Y. and FORTINI, M.E. (1999). Apoptotic activities of wild-type and Alzheimer's disease-related mutant presenilins in Drosophila melanogaster. J. Cell Biol. 146: 1351-1364.
YOUNG, P.E., RICHMAN, A.M., KETCHUM, A.S. and KIEHART, D.P. (1993) Morphogenesis in Drosophilarequires nonmuscle myosin heavy chain function. Genes Dev. 7: 29-41.

ZIMAN, M., O'BRIEN, J.M., OUELLETTE, L.A., CHURCH, W.R. and JOHNSON, D.I. (1991). Mutational analysis of CDC42SC, a Saccharomyces cerevisiae gene that encodes a putative GTP-binding protein involved in the control of cell polarity. Mol. Cell- Biol. 11: 3537-3544.

ZIPKIN, I.D., KINDT, R.M. and KENYON, C.J. (1997). Role of a new Rho family member in cell migration and axon guidance in C. elegans. Cel/90: 883-894.

Received: 21st November 2006 Reviewed by Referees: 18th January 2007 Modified by Authors and Accepted for Publication: 7th May 2007 Published Online: 4th June 2007

\section{Previously published, related Int. J. Dev. Biol. articles of interest}

See our Special Issue on Developmental Genetics of Drosophila edited by Alain Ghysen at: http://www.ijdb.ehu.es/web/contents.php?vol=42\&issue=3

See our forthcoming Special Issue on Developmental Biology in Hispania edited by Juan Arechaga and Maria Carmo-Fonseca at: http://www.ijdb.ehu.es/web/

Developmental regulation of expression of Ran/M1 and Ran/M2 isoforms of Ran-GTPase in mouse testis. Pedro P López-Casas, Luis A López-Fernández, Mario Párraga, Dora B Krimer and Jesús del Mazo Int. J. Dev. Biol. (2003) 47: 307-310

Flamingo, a cadherin-type receptor involved in the Drosophila planar polarity pathway, can block signaling via the canonical wnt pathway in Xenopus laevis.

Richard Morgan, Ali-Morsi El-Kadi and Christopher Theokli

Int. J. Dev. Biol. (2003) 47: 245-252

Cellular polarity, mitotic synchrony and axes of symmetry during growth. Where does the information come from?

D Gubb

Int. J. Dev. Biol. (1998) 42: 369-377

Expression of GTP-binding protein gene drg during Xenopus laevis development. S Kumar, M Iwao, T Yamagishi, M Noda and M Asashima Int. J. Dev. Biol. (1993) 37: 539-546 


\section{Teaching Developmental Biology}

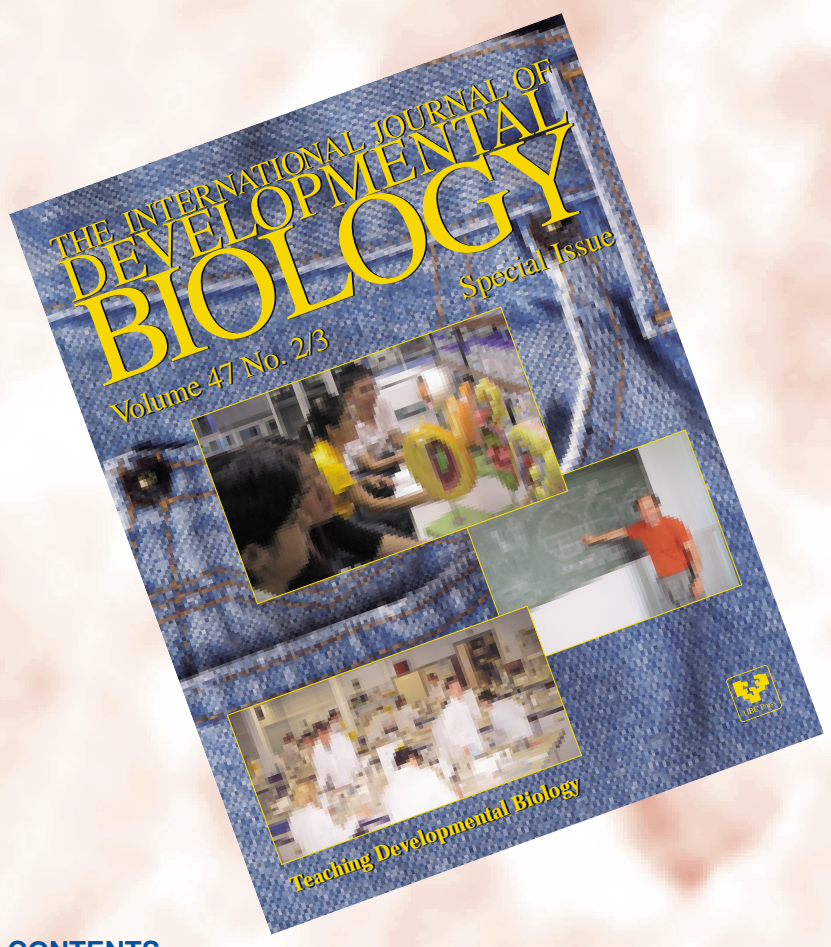

\section{An invaluable resource for researchers with teaching responsabilities!}

\section{CONTENTS}

Introductory Papers

Towards enriching the classroom experience

by George M. Malacinski and Susan T. Duhon

Setting the stage: developmental biology in the precollege classroom

by Sandra Borland, Karen Crawford and Victoria Brand

Examples of Undergraduate Developmental Biology Courses From field to gel blot: teaching a holistic view of developmental phenomena to undergraduate biology students at the University of Tokyo

by Takashi Ariizumi and Makoto Asashima

Integrating developmental biology into the undergraduate curriculum at the University of Bath, United Kingdom by Jonathan M.W. Slack

Making developmental biology relevant to undergraduates in an era of economic rationalism in Australia

by Brian Key and Victor Nurcombe

Learning developmental biology has priority in the life sciences curriculum in Singapore

by Tit-Meng Lim

Developmental biology for undergraduate students at the University of Palermo, Italy

by Giovanni Giudice and Karoly Onorato

Student-oriented learning: an inquiry-based developmental biology lecture course

by George M. Malacinski

Teaching embryology to undergraduates in the Faculty of

Education at Dokuz Eylul University in Izmir, Turkey

by Irfan Yilmaz

Teaching critical thinking in a developmental biology course at an American liberal arts college

by Dany S. Adams

Examples of College Laboratory Courses

Using Xenopus as a model system for an undergraduate

laboratory course in vertebrate development at the University of Bordeaux, France

by Michelle Olive, Pierre Thiebaud, Marc Landry, Michel Duvert, Alain Verna, Wilfrid Barillot and Nadine Theze

The color purple: analyzing alkaline phosphatase expression in experimentally manipulated sea urchin embryos in an undergraduate developmental biology course by Julie Drawbridge

Chick embryo culture techniques employed at Karnatak University in Dharwad, India for studying cellular and molecular aspects of morphogenesis

by Sohan P. Modak

Examples of Advanced and/or Graduate-Level Developmental Biology Courses

An intense half-semester developmental biology course, as taught at Uppsala University, Sweden

by Lennart Olsson

Integrating self-organization theory into an advanced course on morphogenesis at Moscow State University

by Lev V. Beloussov

Reverse engineering the embryo: a graduate course in developmental biology for engineering students at the University of Manitoba, Canada

by Richard Gordon and Cameron A. Melvin

Personal Journeys through Teaching Developmental Biology Developmental biology in Ecuador: a 30-year teaching experience

by Eugenia M. Del Pino

Four decades of teaching developmental biology in Germany by Horst Grunz

My perpetual cycle: from student to researcher to teacher to student...

by Robert Vignali

Course Enhancements and Alternative Learning Strategies Course enhancement: a road map for devising active-learning and inquiry-based science courses

by William $S$. Harwood

The role of textbooks in communicating developmental biology by Leon W. Browder

Using models to enhance the intellectual content of learning in developmental biology

by John C. McLachlan

Virtual labs: a substitute for traditional labs?

by Rebecca K. Scheckler

Broadening the Teaching Agenda beyond Traditional Content Emphases

Educating for social responsibility: changing the syllabus of developmental biology

by Scott F. Gilbert and Anne Fausto-Sterling

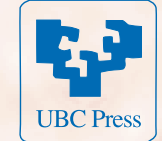

The International Journal of D evelopmental Biology Volume 47 No.2/ 3 (Special Issue) 2003 Order by web at: http:/ / www.ijdb.ehu.es (price 70 US\$ or $€$ ) 